\title{
Teneurin C-terminal associated peptides (TCAP): modulators of corticotropin-releasing factor (CRF) physiology and behavior
}

\author{
Yani Chen, Mei Xu, Reuben De Almeida and David A. Lovejoy*
}

Department of Cell and Systems Biology, University of Toronto, Toronto, ON, Canada

Edited by:

James A. Carr, Texas Tech

University, USA

\section{Reviewed by:}

Cynthia L. Bethea, Oregon Health

and Science University, USA

Vance Trudeau, University of

Ottawa, Canada

*Correspondence:

David A. Lovejoy, Department of Cell and Systems Biology, University of Toronto, 25 Harbord Street,

Toronto, ON M5S 3G5, Canada

e-mail:david.lovejoy@utoronto.ca
The existence of the teneurin C-terminal associated peptides (TCAP) was reported in 2004 after screening a rainbow trout hypothalamic cDNA for corticotropin-releasing factor (CRF)-related homologs. In vertebrates, there are four TCAP paralogs, where each peptide is associated with a teneurin transmembrane protein. The TCAPs are 40 or 41 amino acids in length and possess less than $20 \%$ residue identity with the CRF family of paralogs. Orthologs of TCAP are found in all metazoans with the possible exception of poriferans and cnidarians. Recent evidence indicates that TCAP and the teneurins may have been introduced into the Metazoa via horizontal gene transfer from prokaryotes into a basal protistan. Thus, the origin of the TCAPs likely predated that of the CRF family. In the mammalian brain, TCAP-1 is transcribed independently from teneurin-1. Moreover, TCAP-1 acts on neurons by a CRF-receptor independent signal transduction pathway to regulate cellular cytoskeletal function to stimulate cell activity. Administration of synthetic TCAP-1 to rodents inhibits a number of CRF- and stress-associated behaviors via a hypothalamic-pituitary-adrenal (HPA) axis-independent mechanism.

Keywords: stress, HPA axis, glucocorticoids, dystroglycan, ERK1/2, cytoskeleton, peptide evolution

\section{INTRODUCTION}

Corticotropin-releasing factor (CRF) is part of an evolutionary conserved peptide family integral to the regulation of stressassociated behavior and physiology in metazoans. The long period of time during which CRF-like peptides have persisted suggests that the CRF response has been evolutionarily advantageous. Early-evolving gene systems integral to the survival of an organism are evolutionarily selected for, and because of this, may give rise to additional paralogous lineages via gene, genomic, and chromosomal duplication events. In addition, such early evolving systems will likely become associated with newer evolving molecular and physiological systems. Thus, because of the evolutionary age of the CRF system, it is likely that it is modulated, in turn, by even earlier evolving systems.

One such candidate for an early evolving CRF-modulatory system may be represented by TCAP-1, a member of the teneurin C-terminal-associated peptides family. Highly conserved in all metazoans, TCAP-1 is active in the regulation of metabolism, stress, and reproduction and significantly inhibits a number of CRF-induced stress responses. Although TCAP was originally identified in a rainbow trout cDNA library screen for potential CRF homologs and share a number of structural similarities with the CRF family of peptides, they have less than $20 \%$ sequence similarity to the CRF family (Qian et al., 2004; Lovejoy et al., 2006). In situ experiments in Sprague-Dawley rats show that TCAP is highly expressed in all hippocampal subregions, central amygdala (CeA), basolateral amygdala (BLA), and various hypothalamic nuclei regions that are known to express CRF receptors (Wang et al., 2005). The TCAP-1 peptide also blocks both CRF-induced stress behaviors and c-fos activation within brain areas known to modulate behavioral responses to stress in Wistar rats (Tan et al., 2009). Recent studies show that TCAP exerts its neuromodulatory role on CRF elements independent of the hypothalamic-pituitary-adrenal (HPA) axis, harboring the idea that several accessory pathways may have evolved alongside the HPA axis-mediated signaling pathway.

\section{CRF EVOLUTION AND PHYSIOLOGY}

In vertebrates (Mammalia, Amphibia, Actinoptergyii, and Chondrichthyes), the CRF family consists of four paralogous peptides that are the result of two rounds of genome duplications in early evolution and has been previously described in detail (Lovejoy and Balment, 1999; Lovejoy and Jahan, 2006) (Figure 1). Orthologs of CRF in invertebrates occur as the diuretic hormones in arthropods, and diuretic hormone-like peptide in tunicates. These findings indicate that only a single CRF peptide was present in the genome of an ancestral protochordate. The first of the genome duplications led to the formation of two CRF-related peptides. After subsequent selection, both lineages were retained but modified during the accrual of mutations leading to a modified amino acid sequence. One peptide shares sequence similarities to both CRF and urotensin-1 (urocortin in mammals; Vaughan et al., 1995; Donaldson et al., 1996), whereas the second peptide possesses both urocortin 2 and 3 characteristics (Lovejoy and Jahan, 2006). The second genome duplication led to the formation of the four peptides that are found in extant vertebrates (Lewis et al., 2001; Reyes et al., 2001). However, the presence of CRF orthologs in arthropods indicates that the earliest CRF-like 


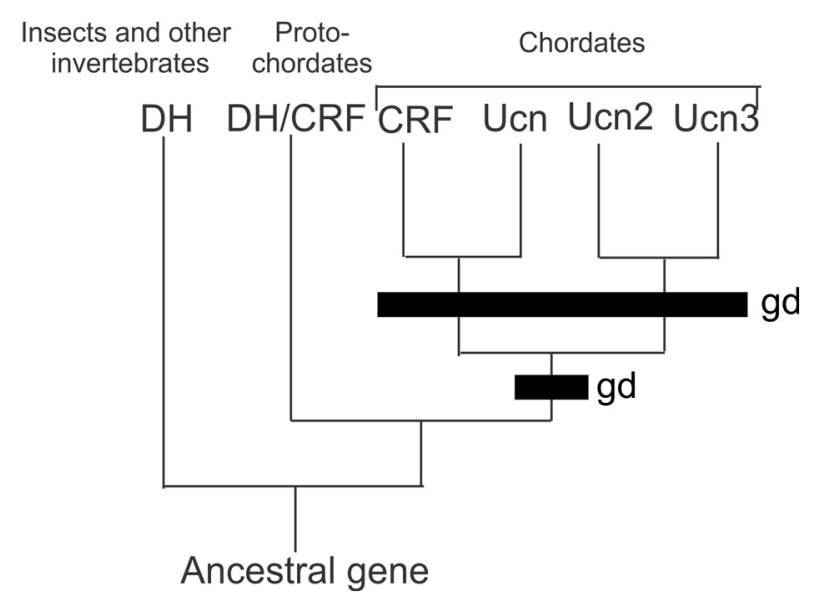

FIGURE 1 | Comparison of the CRF peptides family in chordates by genome duplication. Two rounds of genome duplications early in chordate ancestry gave rise to the four paralogous CRF related peptides.

Abbreviations: $\mathrm{DH}$, diuretic hormone; $\mathrm{DH} / \mathrm{CRF}$, diuretic hormone/corticotrophin releasing factor like peptide; $\mathrm{CRF}$, corticotrophin releasing factor; UCN, urocortin; UCN2, urocortin 2; UCN3 urocortin 3; gd, genome duplication event.

peptides existed before the bifurcation of deuterostome and protostome animals (Lovejoy and Balment, 1999; Lovejoy and Jahan, 2006). Research in the last few decades has provided a basis for understanding the regulation of stress and metabolism by CRF. Currently, it is understood that in vertebrates, CRF activates the stress response through the HPA axis. It is also through this pathway that $\mathrm{CRF}$ is regulated by modulatory components of the central nervous system.

The ancient evolution of CRF and CRF-like peptides indicates that there is functional necessity of the actions of CRF on regulatory mechanisms in physiology. In many species, humans included, CRF induces behaviors and physiological adaptations that endeavor to cope with environmental stressors. Stressors that are associated with new environments, for example, generally bring about behaviors of increased arousal and decreased food intake. Changes in behaviors and physiology that are induced by CRF are mediated by interactions of CRF/Ucn with both the $\mathrm{CRF}_{1}$ and $\mathrm{CRF}_{2}$ receptors. Urocortin-2 and -3 mediate their functions through a separate $\mathrm{CRF}_{2}$ receptor (Hauger et al., 2006).

In numerous vertebrates including Osteichthyes, Amphibia, Reptilia, Avia, and Mammalia species, exposure to stress leads to activation of the hypothalamic-pituitary-adrenocortical (HPA) axis (Barsyte et al., 1999; Lovejoy and Balment, 1999; Lovejoy and Jahan, 2006; Denver, 2009; Lovejoy, 2009). The HPA axis is a neuroendocrine feedback system that is activated when afferents from the sensory system and brainstem signal to the paraventricular nucleus (PVN) to secrete CRF and arginine vasopressin (AVP) hormones into the hypophyseal portal system (Tsigos and Chrousos, 2002; Ulrich-Lai and Herman, 2009). Upon reaching the anterior pituitary, CRF stimulates the corticotropes to secrete adrenocorticotropic hormone (ACTH) into the systemic circulation (Tsigos and Chrousos, 2002; Denver, 2009). This hormone in turn acts on the inner adrenal cortex to synthesize and release glucocorticoids, such as cortisol (Denver, 2009; Ulrich-Lai and Herman, 2009). Circulating glucocorticoids induce a multitude of different responses which all act to promote energy mobilization; these include gluconeogenesis in the liver, liberation of amino acids, inhibition of glucose uptake into muscle and adipose tissue, increased lipolysis and suppression of immune and reproductive functions (Ulrich-Lai and Herman, 2009). Regulation of the system occurs through negative feedback of glucocorticoids at the hypothalamus and anterior pituitary, inhibiting the release of CRF and ACTH respectively; in addition, the action of glucocorticoids on neuronal inputs to the PVN restricts activation of the HPA axis (Tsigos and Chrousos, 2002; Denver, 2009). However, the HPA system, as it is understood in vertebrates, does not appear to be present in non-vertebrates (Lovejoy, 2009). Thus, given the evolutionary age of CRF-like peptides, it has become well ensconced in numerous physiological processes predating the HPA axis. However, CRF itself has a number of non-HPA associated functions including feeding, diuresis, metamorphosis, locomotion, and vocalization (Lovejoy and Balment, 1999). These regulatory actions may have evolved to accompany stress regulation. Given the widespread actions of the CRF family of peptides, evolutionarily older physiological systems may be working alongside the CRF lineage or interacting with elements of the CRF lineage in a neuromodulatory manner in complex organisms.

\section{TENEURIN AND THE TENEURIN C-TERMINAL ASSOCIATED PEPTIDES (TCAP)}

The teneurins are a family of four type-II transmembrane proteins that are critical for morphogenesis and pattern formation in metazoans (Wang et al., 2005; Lovejoy et al., 2006, 2009; Tucker et al., 2007; Young and Leamey, 2009). Originally discovered in Drosophila, teneurins were later discovered in vertebrates to have four paralogs (teneurin-1 to -4) (Kenzelmann et al., 2007). Vertebrate teneurins are predominately expressed in the nervous system and have been implicated in neural development and maintenance (Lovejoy et al., 2006; Kenzelmann et al., 2007). They are typically about 2800 amino acids long with a structure that is highly conserved (Tucker et al., 2007). At the end of the C-terminus of teneurin- 1 to -4 is a cleavable bioactive peptide termed the teneurin C-terminal-associated peptide (TCAP)-1 to -4 . Recent studies, however, indicate that TCAP-1 is independently transcribed from teneurin-1, thus providing evidence for the independence of TCAPs from teneurins (Chand et al., 2012a).

Initial attempts to identify paralogous genes to the CRF family led to the discovery of this TCAP family (Qian et al., 2004). It was first discovered in a screening of the hypothalamic cDNA library of rainbow trout using a hamster urocortin probe (Barsyte et al., 1999; Qian et al., 2004). Sequence analyses revealed a putative peptide sequence on the $3^{\prime}$ region of teneurin- 3 in trout and this peptide sequence was termed TCAP-3, by virtue of its association with teneurin-3 (Qian et al., 2004). Further analyses led to the discovery of the 3 other TCAP peptides on teneurins-1, -2 , and -4 (Wang et al., 2005). The TCAP family is ubiquitously expressed among all metazoan species (Lovejoy et al., 2006; Tucker et al., 2012). The four vertebrate TCAP paralogs show about $80 \%$ identity with each other amongst metazoan species (Lovejoy et al., 
through a PKA/cAMP/CamKII pathway that leads to CREB phosphorylation. Phosphorylated CREB then regulates $c$-fos gene transcription by binding to response elements CRE and AP-1 (Kovacs, 1998). Similarly to CRF, TCAP-1 leads to cAMP accumulation in vitro (Wang et al., 2005). Studies on Gn11 mouse immortalized cell lines show that TCAP-1 has a biphasic effect on cAMP such that low concentrations of TCAP-1 increased cAMP, and high concentrations decreased cAMP levels (Wang et al., 2005). Moreover, a CRF1 receptor antagonist addition failed to block the TCAP-1-induced increase in cAMP suggesting the presence of independent signaling pathways.

Administration of TCAP alone had a minor effect on $c$-fos activation in vivo in rats. However, TCAP administration after concomitant CRF administration produced significant behavioral changes (Tan et al., 2009). This strongly suggests that TCAP plays a neuromodulatory role on elements of CRF signaling systems. Evidence shows that glucocorticoids inhibit AP-1 and CRE responses (Kovacs, 1998). Thus, TCAP could regulate elements of CRF signaling through modulation of CRE and AP-1 activity. In addition, TCAP could inhibit CRF-induced $c$-fos through inhibition of CRF expression itself, as the promoter of the CRF gene contains a CRE (Hauger et al., 2006). Using luciferase reporter assays, we found that TCAP administration decreases AP-1 reporter activity in an immortalized N3 hypothalamic cell line (Nock et al., unpublished observations). Thus, TCAP's ability to attenuate CRF induced $c$-fos activation in vivo may be, in part, through interactions with the AP-1 response element. However, AP-1 is regulated by numerous elements, and TCAP-1 may regulate AP-1 activity by a CRF-independent mechanism.

In vitro, TCAP-1 administration did not alter CRF-induced cAMP increases in the hypothalamic cell line Gn11 (Wang et al., 2005), nor CRF-induced CRE activation in human embryonic kidney cells transfected with $\mathrm{CRF}_{1}$ and $\mathrm{CRF}_{2}$ receptors. In vitro, TCAP-1 does not modulate cellular distribution or total protein of $\mathrm{CRF}_{1}$, or the GC receptors GR and MR in mouse embryonic hippocampal E14 cells. Administration of TCAP-1 also did not modulate the phosphorylation of a $\mathrm{CRF}_{1}$ downstream transcription factor CREB. Repeated daily TCAP-1 administration in mice did not affect basal non-stressed HPA activity based on serum cortisol and ACTH levels. From these studies, it is apparent that under basal non-stressed conditions, TCAP-1 does not interact directly with HPA regulatory pathways involving CRF or GC receptors (De Almeida et al., unpublished studies).

The lack of direct interaction of TCAP-1 on CRF signal transduction systems led us to investigate alternative explanations for its mechanism. The similarity of the TCAP-1 primary structure to CRF and related peptides had led us to postulate that the peptide activated a G-protein coupled receptor (GPCR) similar to the family of GPCRs related to the CRF receptors. However, in vitro screening of most of the receptors in this family did not show any significant binding or activation (Lovejoy, unpublished studies). However, gene microarray profiling indicated a similarity to neurotrophic factor activation and a relationship with the dystroglycan complex (Chand et al., 2012b). Subsequent studies confirmed this relationship, thus TCAP-1 may be the first identified soluble ligand for the dystroglycan complex. The dystroglycan complex is hypothesized to be integral to the intracellular signaling of extracellular TCAP-1 due to a strong co-localization of TCAP- 1 with $\beta$-dystroglycan, the plasma membrane bound subunit of the dystroglycan complex (Chand et al., 2012b). In essence, TCAP-1 mediates cytoskeletal re-organization in E14 cells by binding to the dystroglycan complex which then signals through a MEK- ERK1/2-dependent phosphorylation of stathmin and filamin A. This leads to factin polymerization, increased tubulin immunoreactivity, and increased filopodia development (Chand et al., 2012b). The ERK1/2 pathway is the proposed mechanism through which TCAP-1 modulates neurite and dendrite morphology in immortalized mouse hypothalamic cell lines, primary hippocampal cultures (Al Chawaf et al., 2007a), and Golgi-stained rat brains (Tan et al., 2011).

Within multiple areas of the mouse brain, CRF induces stressrelated cytoskeletal changes. $\mathrm{CRF}_{1}$ activation in the CA3 mediates dendritic retraction by inducing destabilization of $\mathrm{f}$-actin and the cell adhesion molecule nectin-3 (Chen et al., 2008; Wang et al., 2011). Activation of $\mathrm{CRF}_{1}$ in the locus coeruleus (LC) also mediates outgrowth of neuronal processes through the Rho GTPase Rac1 (Swinny and Valentino, 2006). In the hippocampus, this Racl-dependent outgrowth of neuronal processes is counteracted by RhoA inhibition on dendritic spine formation and branching. Thus, TCAP-1 may exert its CRF modulating effects through countering $\mathrm{CRF}_{1}$ mediated changes in cytoskeletal re-organization-like synaptic plasticity within stress modulating brain areas that express both $\mathrm{CRF}_{1}$ and TCAP-1.

\section{CONCLUSION}

Current data suggests that TCAP-1 acts as a separate and distinct peptide modulating system, which is involved in cytoskeletalassociated synaptic plasticity. From an evolutionary perspective, it is perhaps not surprising that TCAP-1 acts independently of CRF signaling. The teneurins and TCAP evolved early in metazoan history, whereas the CRF family of peptides and their role in integrating energy metabolism and the perception of stressors evolved later after evolution of vertebrates (Lovejoy and Jahan, 2006; Lovejoy, 2009). Moreover, the later evolution of the HPA axis in stress signaling and the independence of TCAP-1 neurmodulatory actions from the HPA axis further support a possible parallel evolution. The TCAP-1 peptide acts on the dystroglycan complex in order to signal intracellularly. This signaling may be downstream of other effectors from the CRF signaling pathway downstream of the $\mathrm{CRF}_{1}$ receptor. The observed TCAP-1 modulatory effects on CRF behaviors may then be mediated through TCAP-1 specific signaling that oppose CRF mediated effects on cellular cytoskeletal organization within brain areas that co-express TCAP-1 and CRF. This in turn manifests into modulation of CRF-mediated stress behaviors in brain regions that co-express CRF and TCAP-1.

\section{ACKNOWLEDGMENTS}

We thank NSERC and Protagenic Therapeutics Inc. for funding to carry out this research. Yani Chen and Mei Xu are recipients of NSERC graduate scholarships. 


\section{REFERENCES}

Al Chawaf, A., St. Amant, K., Belsham, D., and Lovejoy, D. A. (2007a). Regulation of neurite growth in immortalized mouse hypothalamic neurons and rat hippocampal primary cultures by teneurin C-terminal-associated peptide-1. Neuroscience 144, 1241-1254. doi: $\quad 10.1016 /$ j.neuroscience.2006. 09.062

Al Chawaf, A., Xu, K., Tan, L., Vaccarino, F. J., and Lovejoy, D. A. (2007b). Corticotropin-releasing factor (CRF)-induced behaviors are modulated by intravenous administration of teneurin Cterminal associated peptide-1 (TCAP-1). Peptides 28, 1406-1415. doi: 10.1016/j.peptides.2007.05.014

Barsyte, D., Tipping, D. R., Smart, D., Conlon, J. M., Baker, B. I., and Lovejoy, D. A. (1999). Rainbow trout (Oncorhynchus mykiss) urotensin-I: structural differences between urotensinsI and urocortins. Gen. Comp. Endocrinol. 115, 169-177. doi: 10.1006/gcen.1999.7290

Chand, D., Casatti, C. A., de Lannoy, L., Song, L., Kollara, A., BarsyteLovejoy, D., et al. (2012a). Cterminal processing of the teneurin proteins: independent actions of a teneurin C-terminal associated peptide in hippocampal cells. Mol. Cell. Neurosci. 52C, 38-50. doi: 10.1016/j.mcn.2012.09.006

Chand, D., Song, L., Delannoy, L., Barsyte-Lovejoy, D., Ackloo, S., Boutros, P. C., et al. (2012b). C-terminal region of teneurin-1 co-localizes with dystroglycan and modulates cytoskeletal organization through an extracellular signal-related kinase-dependent stathmin- and filamin A-mediated mechanism in hippocampal cells. Neuroscience 219, 255-270. doi: 10.1016/j.neuroscience.2012.05.069

Chand, D., de Lannoy, L., Tucker, R., and Lovejoy, D. A. (2013). Origin of chordate peptides by horizontal protozoan gene transfer in early metazoans and protists: evolution of the teneurin C-terminal associated peptides (TCAP). Gen. Comp. Endocrinol. 188, 144-150. doi: 10.1016/j.ygcen.2013.02.006

Chen, Y., Dube, C. M., Rice, C. J., and Baram, T. Z. (2008). Rapid loss of dendritic spines after stress involves derangement of spine dynamics by corticotropin-releasing hormone. J. Neurosci. 28, 2903-2911. doi: 10.1523/JNEUROSCI.022508.2008

de Lannoy, L., and Lovejoy, D. A. (in press). Evolution and phylogeny of the corticotropin-releasing factor (CRF) family of peptides: expansion and specialization in the vertebrates. J. Chem. Neuroanat.

Denver, R. J. (2009). Structural and functional evolution of vertebrate neuroendocrine stress systems. Ann. N.Y. Acad. Sci. 1163, 1-16. doi: $\quad 10.1111 / j .1749-6632.2009$. 04433. $\mathrm{x}$

Donaldson, C. J., Sutton, S. W., Perrin, M. P., Corrigan, A. Z., Lewis, K. A., Rivier, J. E., et al. (1996). Cloning and characterization of human urocortin. Endocrinology 137, 2167-2170. doi: 10.1210/en.137.5.2167

Hauger, R. L., Risbrough, V., Brauns, O., and Dautzenberg, F. M. (2006). Corticotropin Releasing Factor (CRF) receptor signalling in the central nervous system: new molecular targets. CNS Neurol. Disord. Drug Targets 5, 453-479. doi: 10.2174/187152706777950684

Kenzelmann, D., Chiquet-Ehrismann, R., and Tucker, R. P. (2007). Teneurins, a transmembrane protein family involved in cell communication during neuronal development. Cell. Mol. Life Sci. 64, 1452-1456. doi: 10.1007/s00018-007-7108-9

Kovacs, K. J. (1998). c-Fos as a transcription factor: a stressful (re)view from a functional map. Neurochem. Int. 33, 287-297. doi: 10.1016/S0197-0186(98)00023-0

Kupferschmidt, D. A., Lovejoy, D. A., Rotzinger, S., and Erb, S. (2010). Teneurin C-terminal associated peptide-1 blocks the effects of corticotropin-releasing factor on reinstatement of cocaine seeking and on cocaine-induced behavioural sensitization. $\mathrm{Br}$. J. Pharmacol. 162, 574-583. doi: 10.1111/j.1476-5381.2010.01055.x

Lewis, K., Li, C., Perrin, M. H., Blount, A., Kunitake, K., Donaldson, C., et al. (2001). Identification of urocortin III, an additional member of the corticotropin-releasing factor (CRF) family with high affinity for the CRF2 receptor. Proc. Natl. Acad. Sci. U.S.A. 98, 7570-7575. doi: $10.1073 /$ pnas. 121165198

Lovejoy, D. A. (2009). Structural evolution of urotensin-I: retaining ancestral functions before corticotropinreleasing hormone evolution. Gen. Comp. Endocrinol. 164, 15-19. doi: 10.1016/j.ygcen.2009.04.014

Lovejoy, D. A., Al Chawaf, A., and Cadinouche, M. (2006). Teneurin C-terminal associated peptides: an enigmatic family of neuropeptides with structural similarity to the corticotrophin-releasing factor and calcitonin families of peptides. Gen. Comp. Endocrinol. 148, 299-305. doi: 10.1016/j.ygcen.2006.01.012

Lovejoy, D. A., and Balment, R. J. (1999). Evolution and physiology of the corticotropin-releasing factor (CRF) family of neuropeptides in vertebrates. Gen. Comp. Endocrinol. 155, 1-22. doi: 10.1006/gcen.1999.7298

Lovejoy, D. A., and Jahan, S. (2006). Phylogeny and evolution of the corticotropin releasing factor family of peptides. Gen. Comp. Endocrinol. 146, 1-8. doi: 10.1016/j.ygcen.2005.11.019

Lovejoy, D. A., Rotzinger, S., and Barsyte-Lovejoy, D. (2009). Evolution of complementary peptide systems: teneurin $\mathrm{C}$ terminal-associated peptides and corticotropin-releasing factor superfamilies. Ann. N.Y. Acad. Sci. 1163, 215-220. doi: $\quad 10.1111 / \mathrm{j} .1749-6632.2008$. 03629. $\mathrm{x}$

Martins, J. M., Kastin, A. J., and Banks, W. A. (1996). Unidirectional specific and modulated brain to blood transport of corticotropin-releasing hormone. Neuroendocrinology 63, 338-348. doi: 10.1159/000126974

Qian, X., Barsyte-Lovejoy, D., Wang, L., Chewpoy, B., Gautam, N., Al Chawaf, A., et al. (2004). Cloning and characterization of teneurin C-terminal associated peptide (TCAP)-3 from the hypothalamus of an adult rainbow trout (Oncorhynchus mykiss). Gen. Comp. Endocrinol. 137, 205-216. doi: 10.1016/j.ygcen.2004.02.007

Reyes, T. M., Lewis, K., Perrin, M. H., Kunitake, K. S., Vaughan, J., Arias, C. A., et al. (2001). Urocortin II: a member of the corticotropinreleasing factor (CRF) neuropeptide family that is selectively bound by type 2 CRF receptors. Proc. Natl. Acad. Sci. U.S.A. 98, 2843-2848. doi: 10.1073/pnas.051626398

Rotzinger, S., Lovejoy, D. A., and Tan, L. A. (2010). Behavioural effects of neuropeptides in rodent models of depression and anxiety. Peptides 31, 736-756. doi: 10.1016/j.peptides.2009.12.015

Swinny, J. D., and Valentino, R. J. (2006). Corticotropin-releasing factor promotes growth of brain norepinephrine neuronal processes through Rho GTPase regulators of the actin cytoskeleton in rat. Eur. J. Neurosci. 24, 2481-2490. doi: $\quad 10.1111 /$ j.1460-9568.2006. 05129.x

Tan, L. A., Al Chawaf, A., Vaccarino, F. J., Boutros, P. C., and Lovejoy, D. A. (2011). Teneurin C-terminal associated peptide (TCAP)-1 modulates dendritic morphology in hippocampal neurons and decreases anxiety-like behaviors in rats. Physiol. Behav. 104, 199-204. doi: $\quad$ 10.1016/j.physbeh.2011. 03.015

Tan, L. A., Xu, K., Vaccarino, F. J., Lovejoy, D. A., and Rotzinger, S. (2008). Repeated intracerebral teneurin C-terminal associated peptide (TCAP)-1 injections produce enduring changes in behavioral responses to corticotropin-releasing factor (CRF) in rat models of anxiety. Behav. Brain Res. 188, 195-200. doi: 10.1016/j.bbr.2007.10.032

Tan, L. A., Xu, K., Vaccarino, F. J., Lovejoy, D. A., and Rotzinger, S. (2009). Teneurin C-terminal associated peptide (TCAP)-1 attenuates corticotropin-releasing factor (CRF)-induced c-Fos expression in the limbic system and modulates anxiety behavior in male Wistar rats. Behav. Brain Res. 201, 198-206. doi: 10.1016/j.bbr.2009. 02.013

Tsigos, C., and Chrousos, G. P. (2002). Hypothalamic-pituitaryadrenal axis, neuroendocrine factors and stress. J. Psychosom. Res. 53, 865-871. doi: 10.1016/S0022-3999(02)00429-4

Tucker, R. P. (2013). Horizontal gene transfer in choanoflagellates. J. Exp. Zoo. B Mol. Dev. Evol. 320, 1-9. doi: 10.1002/jez.b.22480

Tucker, R. P., Beckmann, J., Leachman, N. T., Scholer, J., and ChiquetEhrismann, R. (2012). Phylogenetic analysis of the teneurins: conserved features and premetazoan ancestry. Mol. Biol. Evol. 29, 1019-1029. doi: 10.1093/molbev/msr271

Tucker, R. P., Kenzelmann, D., Trzebiatowska, A., and ChiquetEhrismann, R. (2007). Teneurins: transmembrane proteins with fundamental roles in development. Int. J. Biochem. Cell Biol. 39, 292-297. doi: 10.1016/j.biocel.2006. 09.012

Ulrich-Lai, Y. M., and Herman, J. P. (2009). Neural regulation of endocrine and autonomic stress and responses. Nat. Rev. Neurosci. 10, 397-409. doi: 10.1038/nrn2647

Vaughan, J., Donaldson, C., Bittencourt, J., Perrin, M. H., Lewis, K., Sutton, S., et al. (1995). Urocortin, a mammalian neuropeptide related to fish urotensin-I and to corticotropin-releasing factor. Nature 378, 287-292. doi: $10.1038 / 378287 \mathrm{a} 0$

Wang, L., Rotzinger, S., Al Chawaf, A., Elias, C. F., Barsyte-Lovejoy, D., Qian, X., et al. (2005). 
Teneurin proteins possess a carboxy terminal sequence with neuromodulatory activity. Mol. Brain Res. 133, 253-265. doi: 10.1016/j.molbrainres.2004.10.019

Wang, X. D., Chen, Y., Wolf, M., Wagner, K. V., and Liebl, C. (2011). Forebrain CRHR1 deficiency attenuates chronic stress-induced cognitive deficits and dendritic remodeling. Neurobiol. Dis. 42, 300-310. doi: 10.1016/j.nbd.2011.01.020

Young, T. R., and Leamey, C. A. (2009). Teneurins: important regulators of neural circuitry. Int. J. Biochem. Cell Biol. 41,
990-993. doi: 10.1016/j.biocel.2008. 06.014

Zhang, D., deSouza, R. F., Anantharaman, V., Iyer, L. M., and Aravind, L. (2012). Polymorphic toxin systems: comprehensive characterization of trafficking modes, mechanism of action, immunity and ecology using comparative genomics. Biol. Direct. 7, 18. doi: 10.1186/1745-61 50-7-18

Conflict of Interest Statement: Dr. D. A. Lovejoy is a co-founder of Protagenic Therapeutics, Inc. The other authors declare that the research was conducted in the absence of any commercial or financial relationships that could be construed as a potential conflict of interest.

Received: 14 May 2013; accepted: 26 August 2013; published online: 17 September 2013.

Citation: Chen $Y, X u$ M, De Almeida $R$ and Lovejoy DA (2013) Teneurin $C$-terminal associated peptides (TCAP): modulators of corticotropin-releasing factor (CRF) physiology and behavior. Front. Neurosci. 7:166. doi: 10.3389/ fnins.2013.00166
This article was submitted to Neuroendocrine Science, a section of the journal Frontiers in Neuroscience. Copyright (C) 2013 Chen, Xu, De Almeida and Lovejoy. This is an open-access article distributed under the terms of the Creative Commons Attribution License (CCBY). The use, distribution or reproduction in other forums is permitted, provided the original author(s) or licensor are credited and that the original publication in this journal is cited, in accordance with accepted academic practice. No use, distribution or reproduction is permitted which does not comply with these terms. 